Final Progress Reports: DE-FG02-02ER45975 University of Maryland \& DE-FG02-02ER45976 Purdue University

\title{
Project Title: Virus Assemblies as Templates for Nanocircuits
}

\author{
Project Directors: \\ James N Culver \\ Univ. of Maryland \\ Institute for Biosciences \& Biotechnology \\ College Park MD 20742-4450 \\ DE-FG02-02ER45975(Culver)
}

\author{
Michael T Harris \\ Purdue University \\ School of Chemical Engineering \\ West Lafayette, IN 47907-2100 \\ DE-FG02-02ER45976 (Harris)
}

Period Covered: $\quad$ 7/01/08 to $6 / 30 / 11$

\section{Summary:}

The goals of this project were directed at the identification and characterization of biomineralization processes and patterning methods for the development of nano scale materials and structures with novel energy and conductive traits. This project utilized a simple plant virus as a model template to investigate methods to attach and coat metals and other inorganic compounds onto biologically based nanotemplates. Accomplishments include: the development of robust biological nanotemplates with enhanced inorganic coating activities; novel coating strategies that allow for the deposition of a continuous inorganic layer onto a bio-nanotemplate even in the absence of a reducing agent; three-dimensional patterning methods for the assemble of nano-featured high aspect ratio surfaces and the demonstrated use of these surfaces in enhancing battery and energy storage applications. Combined results from this project have significantly advanced our understanding and ability to utilize the unique self-assembly properties of biologically based molecules to produce novel materials at the nanoscale level.

\section{Specific Accomplishments:}

Patterned DNA assembled TMV structures. Studies in this objective have focused primarily on patterning TMV based templates into controlled positions via nucleic acid methodologies. Previously we developed a procedure for the patterning of TMV templates via nucleic acid hybridization. Specifically the partial exposure of the viral 5' end genome sequence can be used to pattern virus templates onto silica chips or glass slides containing patterned probe DNA. This patterning technique represents a significant advancement toward developing TMV as a bio-template for use in creating nano-circuits and devices. As a means to produce more complex nanoscale structures we have assemble DNA ladders to produce chains
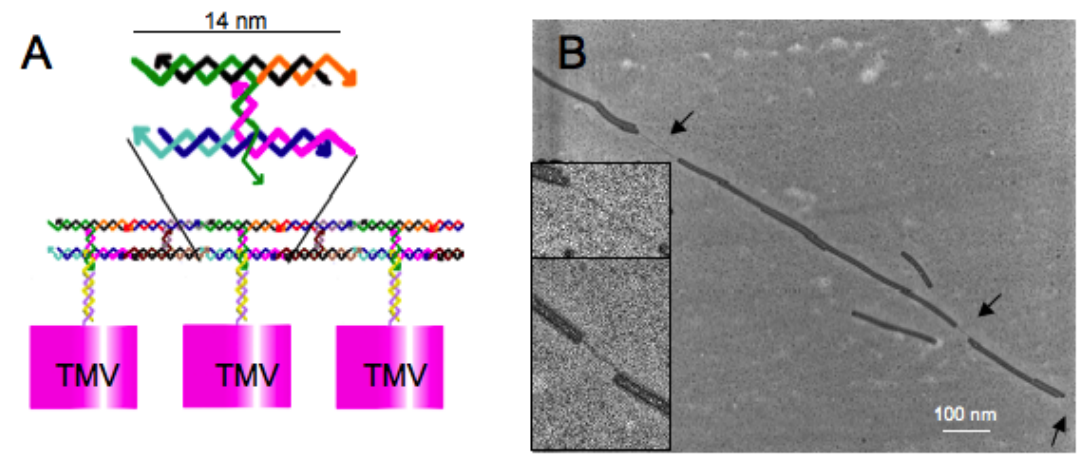

Figure 1. DNA ladder directed assembly of TMV nanowires. A, Diagram of DNA ladder assembly with TMV binding sites positioned at every other rung. Each individual DNA segment that makes up the ladder is highlighted in a different color. B, Electron micrograph displaying a DNA assembled virus nanowire. The exposed DNA ladder is visible at three locations along the wire, marked by arrows. 
of the virus templates (Fig. 1). Using 12 nucleotide long DNA oligomers we assembled DNA ladders composed of double stranded DNA with individual rungs separated by $14 \mathrm{~nm}$ and rails separated by $7 \mathrm{~nm}$. Our present design includes a TMV specific DNA address sequence located at every other rung. DNA ladders are self-assembled in one-pot reactions, followed by hybridization in solution to partially disassembled TMV particles with exposed 5' nucleic acid sequences. Results indicate that DNA ladders readily assembly and can function to chain TMV particles together along the length of the ladder (Fig. 1).

Development of a novel reducing agent free mineralization process. One of the main goals of our studies has been to develop strategies for the uniformed coating of nano-templates with various reactive metals. In previous efforts we used a novel combination of genetically engineered viruses and electroless platting techniques to obtain near uniform nickel and cobalt coatings on patterned virus templates. However, controlling the inorganic mineralization on nano-biotemplates over the entire sample during the solution process has proven difficult. The use of excessive amounts of the inorganic precursor such as palladium chloride in combination with reducing chemicals such as hydrazine and dimethylamine borane generally resulted in uncontrollable and non-templated metal deposition. To eliminate these inefficiencies we developed a new synthetic strategy for the controlled aqueous-phase palladium crystallization of nano-biotemplates. This low cost, solution processing method yields continuous and uniform coatings of palladium on TMV, creating palladium nanowires of tens of nanometers in thickness and hundreds of nanometers in length. This approach utilizes a palladium chloride precursor to produce metallic Pd coatings on TMV without the need for an external reducing agent (Fig. 2).

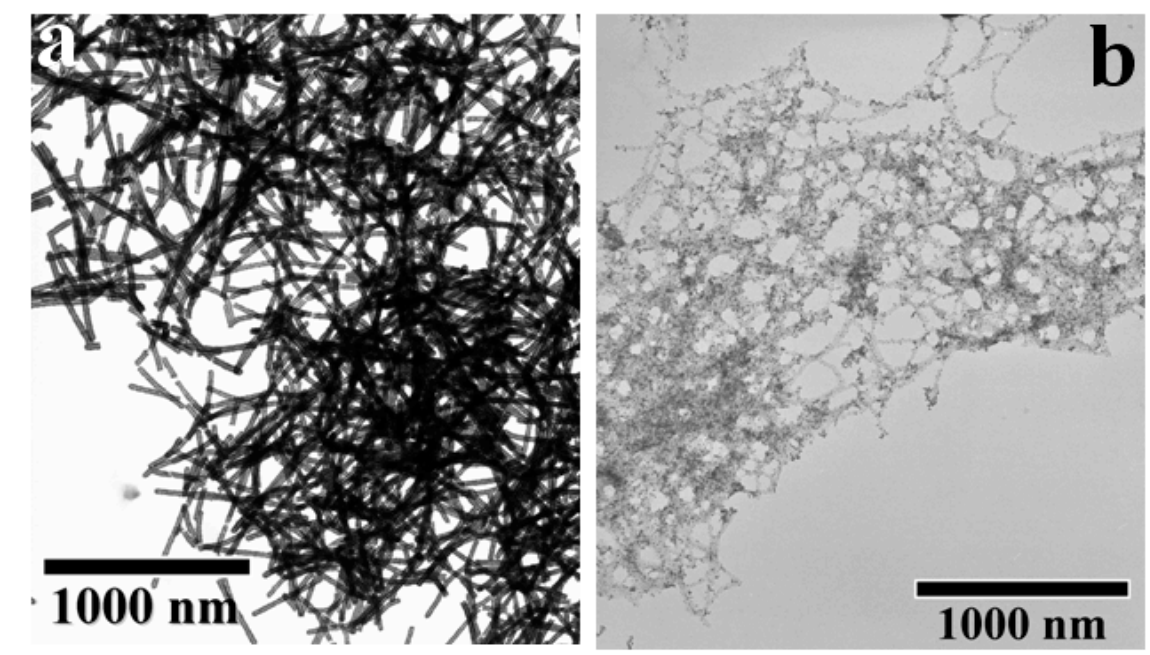

Figure 2. Comparison of $\mathrm{Pd}$ mineralization on the TMV template using a) self-mineralization process without reducing agent and b) standard electroless plating process with the addition of the reducing agent DMAB.

Biosorption capacity of metal precursors on TMV. We also investigated the relationship between the loading of metal precursor ions for $\mathrm{Au}(\mathrm{III})$ and $\mathrm{Pd}$ (II) and the overall metal deposition onto the genetically-modified model biological template, TMV1cys. The loading of the metal precursor ions was measured as a function of the equilibrium concentration of $\mathrm{Pd}(\mathrm{II})$ or $\mathrm{Au}(\mathrm{III})$ at several temperatures. The Langmuir isotherm was used to model the initial loading of the ions on the TMV particle. However, further studies indicated that the $\mathrm{Pd}(\mathrm{II})$ and $\mathrm{Au}(\mathrm{III})$ ions were eventually bound to the TMV irreversibly. Our investigation showed an improvement of the loading of $\mathrm{Pd}(\mathrm{II})$ precursor ions on TMV1Cys in comparison to the unmodified wild-type TMV. Furthermore, the Pd coating on TMV increased with the loading capacity of the metal precursor ion on the TMV. This indicated that the Pd particles most probably nucleated on the surface of the TMV. 
Modeling the controlled deposition of TMV. Solution of the governing transport phenomena equations using the Galerkin finite element method has been used to study the deposition of spherical colloidal particles on a flat substrate during the evaporation of a sessile drop. This algorithm represents the first step in the development of models to predict the deposition pattern of TMV, an asymmetric colloidal particle, during the evaporation of a sessile drop in the absence of an electric field and ultimately in the presence of an electric field. The operating diagram for the deposition pattern of the spherical colloidal particles during the evaporation of a sessile drop was computed as a function of dimensionless groups, the Damkholer number and Pectlet number.

Silica stabilized virus templates. The use of biological molecules as templates for the production of metal nanoparticles and wires is sometime limited by the stability of the bio-template and its affinity for nucleating metal deposition, particularly at high metal ion concentrations and in non-aqueous solvent systems. We used TMV to investigate the use of silica coatings as a means to both enhance template stability and increase its affinity for metal ions. Results from this study indicate that the unmodified TMV particle can function as a template for the growth of thin $(<1 \mathrm{~nm})$ silica layers. However, this thin silica shell did not enhance the stability of the template during metal deposition in organic solvent systems. To increase silica growth on the TMV template, a pretreatment

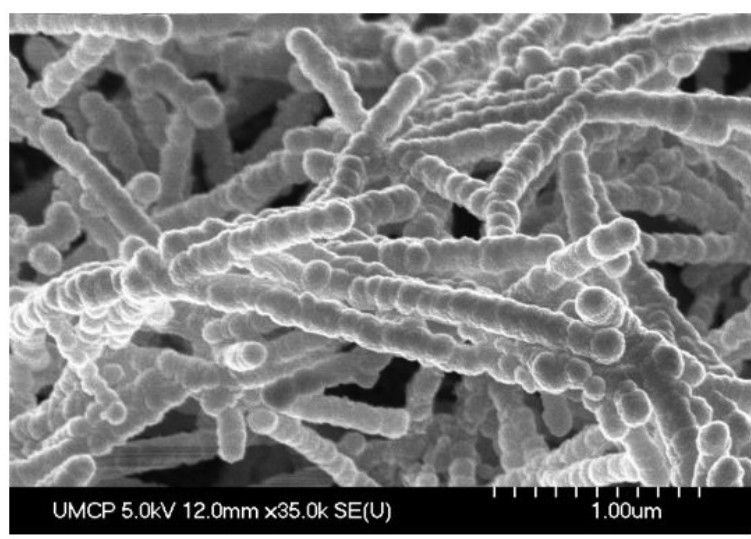

Figure 3. Surface assembled TMV particles coated with silicon. These surfaces can be subsequently coated with a variety of inorganics as described. strategy using aniline was used to produce a uniform silica attractive surface. Aniline pretreated templates yielded significant silica layers of $>20 \mathrm{~nm}$ in thickness (Fig. 3). These silica shells conferred a high degree of stability to the TMV particle and promoted the deposition of various metal nanoparticles through conventional silica mineralization chemistries. This process increased the stability of the TMV template for the deposition of metals at high densities and in organic solvents and also promotes the long term storage, sonication stability and resistance to drying effects.

Surface and conductive properties of hierarchal assembled virus structures. Using the known threedimensional structure of TMV we created a novel mutant, TMV1cys, by inserting a cysteine codon within the $\mathrm{N}$-terminus of the coat protein open reading frame. The positioning of the 1 cys mutation directs the attachment and vertical positioning of the viral rod shaped particles onto gold, steel, or other metal surfaces (Fig. 4). Although surface exposed, the 1cys mutation is recessed within a groove and partially covered by the C-terminal arm of the coat protein. This position inhibits direct contact between the cysteine derived thiol and metal surfaces except at the 3 ' end of the virion rod where the thiol group is sufficiently exposed to make direct contact with the gold surface. This vertical positioning combined with our electroless plating methods produces

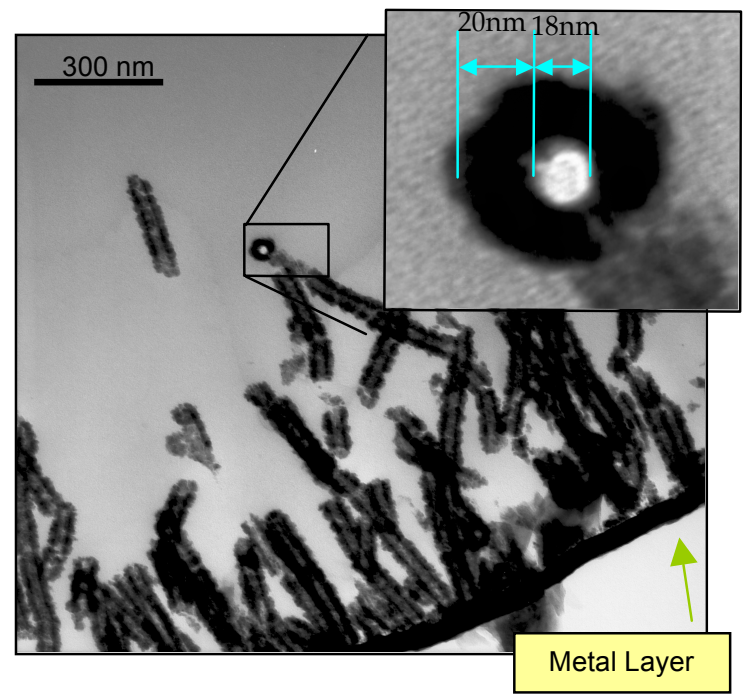

Figure 4. Vertical assembly and Ni coating of TMV1cys particles onto a metal surface. evenly coated virus templated surfaces containing confluent metal coatings as thin as $15 \mathrm{~nm}$ in thickness. 
In addition, the perpendicular assemble of the virus particles significantly increasing available surface area by $>80$ fold.
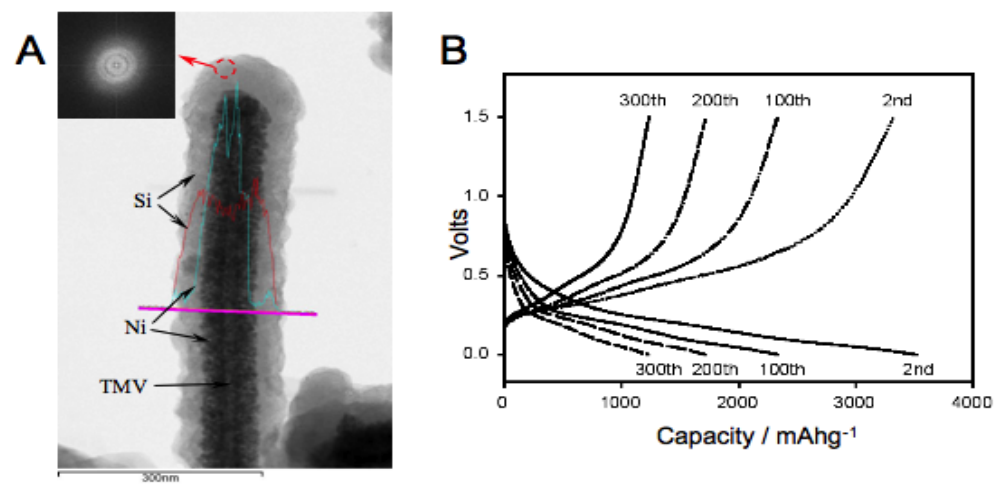

Figure 5. TMV templated Si electrode. A, TEM with EDS spectra and FFT image confirming the architecture of the anode. $\mathrm{B}$, Charge-discharge profile of the anode at the $2^{\text {nd }}, 100^{\text {th }}, 200^{\text {th }}$, and $300^{\text {th }}$ cycle.

We have since investigated these surface assembled virus templates as electrodes. In our initial studies we used a simple NiO-Zn battery system to examine electrode activity. These TMV-assembled $\mathrm{NiO}$ electrodes consistently out-performed non TMV-templated electrodes by a order of magnitude. More recently we have developed a silicon - Li ion anode electrode using the TMV self-assembly process (Fig. 5). Silicon has emerged as one of the most promising next-generation anode materials for lithiumion batteries due to its high theoretical capacity of $3572 \mathrm{mAhg}^{-1}$ per weight and $8322 \mathrm{mAh} / \mathrm{cm}^{3}$ per volume for $\mathrm{Li}_{3.75} \mathrm{Si}$ at room temperature. However, $\mathrm{Si}$ has not been widely utilized in battery systems because of the extreme volume change, 300 400\%, that occurs during lithium insertion and extraction, resulting in pulverization of the anode material and loss of connectivity to the current collector. Recently, the architecture of silicon nano-wires and nano-tubes has been shown to produce stable silicon anodes that can accommodate the volume changes that accompany silicon charge and discharge cycles. We have combined virus assembly and patterning with electroless chemistries and vapor deposition to produce a novel silicon based anode architecture that displays enhanced capacity $\sim 1100 \mathrm{mAh} / \mathrm{g}$ over extended charge - discharge runs, $>300$ cycles. The capacity of this virus enabled anode is $\sim 3$ times the typical capacity $(250-350 \mathrm{mAh} / \mathrm{g})$ of current commercially available graphite based anodes. This capacity level can be attributed to the unique architecture of the anode with each Si nanorod containing a nickel coated virus that functions as a highly conductive matrix connecting the Si surface directly to the steel current collector and to the $\sim 80$ fold increase in surface area afforded by the self-assembled virus surface.

\section{Publications Citing Award:}

Royston, E, Ghosh, A, Kofinas P, Harris MT, and Culver JN. 2008. Self-assembly of virus structured high surface area nano-materials and their application as battery electrodes. Langmuir 24:906-912.

Widjaja E, and Harris MT. 2008 Numerical Study of Vapor Phase-Diffusion Driven Sessile Drop Evaporation. Computers \& Chemical Engineering, 32:2169-2178.

Widjaja E, and Harris MT. 2008. Particle Deposition Study during Sessile Drop Evaporation. AIChE J, 54:2250-2260.

Lee SY, Lim JS, Culver JN, Harris MT. 2008. Coagulation of tobacco mosaic virus in alchohol-waterLiCl solutions. J. Colloid Interface Sc.i 324:92-8.

Royston ES, Brown AD, Harris MT, Culver JN. 2009 Preparation of silica stabilized Tobacco mosaic virus templates for the production of metal and layered nanoparticles. J. Colloid Interface Sci. 332:402-407.

Gerasopoulos K, McCarthy M, Banerjee P, Fan X, Culver JN, Ghodssi R. 2010. Biofabrication methods for the patterned assembly and synthesis of viral nanotemplates. Nanotechnology 21:55304. 
Lim JS, Kim SM, Lee SY, Stach EA, Culver JN, Harris MT. 2010. Quantitative study of Au(III) and $\operatorname{Pd}(\mathrm{II})$ ion biosorption on genetically engineered Tobacco mosaic virus. J. Colloid Interface Sci. 342:455-61.

Lim JS, Kim SM, Lee SY, Stach EA, Culver JN, Harris MT. 2010. Formation of Au/Pd alloy nanoparticles on TMV. J. Nanomaterials. Article \#620505.

Chen X, Gerasopoulos K, Guo J, Brown A, Wang C, Ghodssi R, Culver JN. 2010. Virus-enabled silicon anode for lithium-ion batteries. ACS Nano 4:5366-72.

Lim JS, Kim SM, Lee SY, Stach EA, Culver JN, Harris MT. 2010. Biotemplated aqueous-phase palladium crystallization in the absence of external reducing agents. Nano Lett. 13;10(10):3863-7.

Chen X, Gerasopoulos K, Guo J, Brown A, Wang C, Ghodssi R, Culver JN. 2011. A patterned silicon anode fabricated by electrodeposition of $\mathrm{Si}$ on a virus structured 3-dimensional current collector, Advanced Functional Materials, 21:380-87.

Chen X, Gerasopoulos K, Guo J, Brown A, Ghodssi R, Culver JN, Wang C. 2011. High rate performance of virus enabled 3D n-type Si anodes for lithium-ion batteries. Electrochimica Acta 56:5210-13.

Lim JS, Kim SM, Lee SY, Stach EA, Culver JN, Harris MT. 2011. Surface functionalized silica as a toolkit for studying aqueous phase palladium absorption and mineralization on thiol moiety in the absence of external reducing agents. J. Colloid Interface Sci 356:31-36.

Project Personnel:

Purdue Laboratory:

Dr. Mike Harris

Jung Sun Lim

Pei-Fang Sung

Maryland Laboratory

Dr. James Culver

Adam Brown

Dr. Lisa Parsons

\section{Percent Support:}

PI

Graduate Student

Graduate Student

$5 \%$

$100 \%$

$50 \%$

PI

Research Associate $\quad 50 \%$

Post-Doctoral Associate $\quad 100 \%$

Jung Sun Lim was a graduate student working on the project in Dr. Harris's Laboratory at Purdue University. He concentrated on fundamental experiments to elucidate the mechanism by which the particles form on the biotemplate. He has also spent time in Dr. Culver's laboratory at the University of Maryland learning techniques for the purification and the patterning of TMV. Ms. Pei-Fang Sung is a current graduate student in Dr. Harris laboratory and worked on the development of computer algorithms to model the alignment and deposition patterns of the TMV bio-nanorod on a smooth substrate. Dr. Parsons was post-doctoral student in Dr. Culver's laboratory and worked on DNA patterning efforts. Adam Brown is the research associate in Dr. Culver's laboratory and was responsible for the production of virus mutants and the development of metal coating strategies. This project provided significant crossdisciplinary training to participating personnel, covering the fields of biology, protein engineering and materials science. 